\title{
Is there Nuclear Pinning of Vortices in Superfluid Pulsars?
}

\author{
Paola Donati and Pierre M. Pizzochero \\ Dipartimento di Fisica, Università degli Studi di Milano, and Istituto Nazionale di Fisica Nucleare, sezione di Milano, \\ Via Celoria 16, 20133 Milano, Italy
}

(Received 22 October 2002; published 30 May 2003)

\begin{abstract}
We develop a fully consistent semianalytical model in order to study the vortex-nucleus interaction in the inner crust of neutron stars. In the framework of the local-density approximation and assuming a constant pairing gap and a square-well nuclear potential, the model takes into account all energy contributions and determines unambiguously the structure of the vortex core. The results show that, irrespective of the value of the pairing gap, only interstitial pinning takes place all along the inner crust. This is in contrast with all existing calculations, which predict nuclear pinning in the deeper layers of the crust. Should further studies confirm this surprising result, the explanation of pulsar glitches in terms of depinning of vortices will have to be carefully revisited.
\end{abstract}

DOI: 10.1103/PhysRevLett.90.211101

Rotating neutron stars (pulsars) have been observed to undergo sudden spin-ups in their rotational frequencies; these "glitches" may be connected to nucleon superfluidity [1]. Indeed, the neutron superfluid permeating the inner crust cannot follow the pulsar's rigid rotation, since superfluid flow must be irrotational. Instead, it develops an array of microscopic linear vortices, each carrying angular momentum. A vortex corresponds to a velocity field $\vec{v}$ which is singular on the vortex line (axis), but preserves the condition $\vec{\nabla} \times \vec{v}=0$ everywhere else. According to the model [1], the vortex lines are pinned (bound) to the lattice of nuclei present in the inner crust. Under certain conditions, the vortices can be unpinned from the lattice and deliver their angular momentum to the star's surface, thence spinning it up. To date, all predictions for pulsar glitches based on the vortex model rely on a very limited set of results for the crucial pinning energies [2-4]. These are defined as the difference in energy between two competing configurations, one with the vortex line positioned on a nucleus (nuclear pinning), the other with the vortex line equidistant between two nuclei (interstitial pinning). The problem is how to calculate the energies of these configurations in a physically reliable way, and thence determine which kind of pinning is energetically favored along the inner crust.

Existing studies all agree in predicting nuclear pinning deep inside the inner crust, for densities larger than $\sim 10^{13} \mathrm{~g} / \mathrm{cm}^{3}$, but they yield quite different values for the pinning energies in these regions. The first rough estimates [2] considered only the difference in pairing condensation energy between the two vortex-nucleus configurations, evaluated in a crude model with uniform densities for both nuclear and neutron matter. It took more than ten years [3] to recognize two crucial issues, namely, that differences in kinetic energy had to be considered in addition to those in condensation energy and that the peculiar density profile of the Wigner-Seitz (WS) cells, determined for the inner crust of neutron stars [5], had to be taken into account. The calculations
PACS numbers: $26.60 .+\mathrm{c}, 97.60 . \mathrm{Gb}$

[3] are based on the Ginzburg-Landau theory to evaluate the pairing properties of the neutron superfluid. The conditions of applicability of the theory, however, are far from being satisfied in the inner crust and the authors must artificially rescale the Ginzburg-Landau results by large factors, in order to reproduce the pairing properties of ordinary nuclei. A more satisfying approach was presented ten years later [4], using the Thomas-Fermi model based on the local-density approximation (LDA) to describe the pairing properties of the system. The rationale behind this choice is that, although unable to describe correctly the density tail of the system, where the density goes to zero, the LDA is expected to yield reasonable results for quantities averaged over the whole system such as the energies, which are integrated over the WS cell. These authors [4] also obtained nuclear pinning at large densities, but with pinning energies about 1 order of magnitude lower than the ones obtained before, a fact which tends to improve the agreement between theory and observations. The difficulty of performing a quantum calculation for the complicated geometries of the vortexnucleus configurations suggests that, in spite of its known limitations, the LDA approach is to date the most satisfying one. When applied to the thermal properties of the inner crust of neutron stars, the LDAyields results that are qualitatively compatible with those from the complete quantum calculation, albeit quantitatively different as a consequence of proximity effects due to the nonlocal nature of pairing [6].

The work of Ref. [4], however, although indicating a convenient approach to evaluate the vortex-nucleus interaction, eventually failed to develop the model consistently by making some assumptions that we now see as unjustified and basically incorrect. There are four points in Ref. [4] we are now critical about. The first is the definition of the vortex core which, as done in all previous works, was taken as the superfluid coherence length $\xi$, namely, the distance from the vortex axis where the increase of kinetic energy, due to the vorticous rotation 
of superfluid neutrons, equals the decrease in condensation energy, due to the pairing of neutrons in Cooper pairs. The rationale behind this choice was that inside this radius it is energetically more convenient for matter to be in a normal state and at rest in a frame comoving with the star normal component (e.g., the nuclear lattice), while outside of $\xi$ matter is superfluid and moving irrotationally around the vortex axis with the (negative) condensational term dominating the kinetic one. Such a prescription, however, is inconsistent with the ThomasFermi model, which describes the hydrostatic equilibrium under degeneracy pressure of a Fermion gas subject to position-dependent potentials. Indeed, the normal and superfluid phases should coexist in equilibrium at $r=\xi$, while it is easy to show that in the Thomas-Fermi model the pressures of the two phases are different at that point, thus making hydrostatic equilibrium between them impossible. A second criticism concerns the structure of the vortex itself. Indeed, based on the previous energy argument, the vortex core has always been taken as consisting of normal matter. However, one should also consider the possibility that the core is empty of matter, while still preserving hydrostatic equilibrium. Of the two scenarios, the system will choose the one with minimum total energy [7]. The third objection is that in Ref. [4] only the condensational and kinetic contributions to the energy were taken into account, neglecting the internal energy terms. These are density dependent and since the LDA approach yields different density profiles for the two pinning configurations, one expects differences also in the internal energies. The last criticism concerns the evaluation of the kinetic terms, which was not made in a consistent way. Indeed, for the nuclear pinning configuration they were calculated directly in the LDA, while for the interstitial pinning case an analytical expression derived in Ref. [3] was used, which assumes constant densities and is thus at odds with the LDA.

In this Letter we give a satisfying answer to the previous objections, by developing a fully consistent semianalytical schematic model to study the vortex-nucleus interaction, which describes the presence of vortex lines in the neutron superfluid that permeates the lattice of neutron-rich nuclei in the inner crust of neutron stars. In the framework of the local-density approximation and assuming a constant pairing gap and a square-well nuclear potential, the model takes consistently into account all energy contributions (condensational, kinetic, and internal). It also determines unambiguously the radius of the vortex core which, depending on the density and on the vortex-nucleus configuration, turns out to be either small and empty of matter or large and made of normal matter. The results show that, irrespective of the value of the pairing gap, only interstitial pinning takes place all along the inner crust. This is in contrast with all existing calculations, which predict nuclear pinning in the deeper regions of the inner crust, the difference arising from both the structure of the vortex core and the inclusion of the internal energy contribution. Should further studies that also go beyond the present semiclassical treatment of pairing confirm this surprising result, the explanation of pulsar glitches in terms of depinning of vortices from the nuclear lattice will have to be carefully revisited.

We now briefly describe our physical model. The microscopic structure of the inner crust of neutron stars was studied in Ref. [5] for different average baryon densities $\rho$, in terms of spherical WS cells of radius $R_{\mathrm{WS}}$ containing $N$ neutrons and a central nucleus of radius $R_{N}$, with $Z$ protons and $N_{\text {bound }}$ bound neutrons [8]. The gas of unbound neutrons is characterized by a number density $n_{G}$ (outside of the nucleus) and their number in each cell is $N_{\text {free }}^{\prime}=N-N_{\text {bound. }}$. In Table I we report the parameters associated with four zones along the inner crust. Since we will consider vortex configurations involving two WS cells, in our model we will take cubic cells with side $2 R_{\mathrm{WS}}$ rather than spherical ones, in order to calculate consistently the energy differences. The number of unbound neutrons must then be renormalized to $N_{\text {free }}=$ $6 N_{\text {free }}^{\prime} / \pi$.

Since the crust temperature is low compared to the typical critical temperatures of pairing, we will assume $T=0$. Then, in the framework of the LDA, we define the neutron local Fermi momentum $k_{f}(\mathbf{x})$ related to the number density of neutrons $n(\mathbf{x})$ by the usual expression $3 \pi^{2} n(\mathbf{x})=k_{f}^{3}(\mathbf{x})$. The Thomas-Fermi method consists of occupying energy levels up to the Fermi energy $\mu$ which, at any given $\rho$, is constant throughout the WS cells. We thus impose the constraint $\mu=\sum_{i} \mu_{i}(\mathbf{x})$, where the sum is over $i=\{$ Fermi, nuclear, kinetic, condensation $\}$ for the superfluid phase and $i=\{$ Fermi, nuclear $\}$ for the normal phase [9]. The nuclear term in our schematic model is that of a spherical potential box of depth $U_{0}$ and radius $R_{N}$; that is $\mu_{\text {nuc }}(\mathbf{x})=-U_{0} \theta\left(r-R_{N}\right)$, with $r=|\mathbf{x}|$ the distance from the center of the nucleus. The Fermi term has the standard expression $\mu_{\text {fer }}(\mathbf{x})=\hbar^{2} k_{f}^{2}(\mathbf{x}) / 2 m_{n}$, with $m_{n}$ the neutron mass. The kinetic term due to the irrotational motion around the vortex axis is $\mu_{\mathrm{kin}}(\mathbf{x})=\hbar^{2} / 8 m_{n} R^{2}$, with $R$ the distance from the vortex axis. Finally,

TABLE I. Physical parameters of the four zones in the inner crust. The values are taken from Ref. [5]. The average baryon densities, $\rho$, are given in $\mathrm{g} / \mathrm{cm}^{3}$, the densities of the free neutron gas, $n_{G}$, in $\mathrm{fm}^{-3}$, the radii of the nuclei, $R_{N}$, and those of the WS cells, $R_{\mathrm{WS}}$, in fm. The total number of neutrons in each cell and the number of protons and neutrons bound in nuclei are indicated by $N, Z$, and $N_{\text {bound }}$, respectively.

\begin{tabular}{ccccc}
\hline \hline Zone & 1 & 2 & 3 & 4 \\
\hline$\rho$ & $1.5 \times 10^{12}$ & $9.6 \times 10^{12}$ & $3.4 \times 10^{13}$ & $7.8 \times 10^{13}$ \\
$n_{G}$ & $4.8 \times 10^{-4}$ & $4.7 \times 10^{-3}$ & $1.8 \times 10^{-2}$ & $4.4 \times 10^{-2}$ \\
$R_{N}$ & 6 & 6.7 & 7.3 & 6.7 \\
$R_{\mathrm{WS}}$ & 44 & 35.5 & 27 & 19.4 \\
$N$ & 280 & 1050 & 1750 & 1460 \\
$Z$ & 40 & 50 & 50 & 40 \\
$N_{\text {bound }}$ & 110 & 110 & 110 & 70 \\
\hline \hline
\end{tabular}


TABLE II. Physical parameters of the four zones in the inner crust calculated in the semiclassical model with a constant pairing gap $\Delta=1 \mathrm{MeV}$. The number of unbound neutrons, renormalized to a cubic cell of side $2 R_{\mathrm{WS}}$, is indicated by $N_{\text {free }}$. The depth of the nuclear potential, $U_{0}$, and the chemical potential, $\mu$, are given in $\mathrm{MeV}$. The energy terms for a cubic WS cell without vortex are reported in $\mathrm{MeV}$.

\begin{tabular}{ccccc}
\hline \hline Zone & 1 & 2 & 3 & 4 \\
\hline$N_{\text {free }}$ & 324 & 1795 & 3132 & 2654 \\
$U_{0}$ & 48.67 & 38.68 & 32.70 & 28.70 \\
$\mu$ & 1.10 & 5.74 & 14.40 & 24.92 \\
$E_{0, \text { fer }}$ & 3692.8 & 9718.2 & 31755.2 & 43823.9 \\
$E_{0, \text { nuc }}$ & -5536.5 & -5235.7 & -6218.5 & -5129.2 \\
$E_{0, \text { con }}$ & -101.2 & -116.2 & -80.9 & -39.6 \\
$E_{0, \text { tot }}$ & -2007.9 & 4366.3 & 25455.7 & 38655.2 \\
\hline \hline
\end{tabular}

the pairing condensation energy is $\mu_{\text {con }}(\mathbf{x})=-\Delta^{2} m_{n} /$ $4 \hbar^{2} k_{f}^{2}(\mathbf{x})$, with $\Delta$ the pairing gap, taken as a constant input parameter in our model.

The Thomas-Fermi ansatz $\mu=\sum_{i} \mu_{i}(\mathbf{x})$ determines analytically the neutron density $n(\mathbf{x})$ as a function of position in the cell, for either the normal phase or the superfluid one, once the Fermi energy $\mu$ is known. Since, according to the Feynman-Onsager relation, the mean spacing between vortex lines is orders of magnitude larger than $R_{\mathrm{WS}}$ for even the fastest pulsars, we can take the vortices as isolated. The $1 / R^{2}$ behavior of the kinetic term guarantees that the effect of the vortex becomes negligible as one moves away from the vortex axis. Therefore, the vortex is surrounded by regular (without vortex) superfluid cells, which determine the constant value of $\mu$. In other words, the surrounding cells act as a particle bath, which exchange neutrons with the vortex region in such a way as to maintain $\mu$ fixed.

In Table II we give the results of our model for a vortexfree [i.e., $\mu_{\text {kin }}(\mathbf{x}) \equiv 0$ ] cubic WS cell with pairing gap $\Delta=1 \mathrm{MeV}$. The parameters $U_{0}$ and $\mu$ are determined for each zone in order to give the correct number of bound and unbound neutrons, which can be obtained by integrating $n(\mathbf{x})$ over the entire cell. Once the nuclear well depth and the Fermi energy are found, it is possible to calculate the total energy of the cell $E_{0, \text { tot }}=\sum_{i} E_{0, i}$ by integrating over its volume the total energy density $\epsilon_{\mathrm{tot}}(\mathbf{x})=\sum_{i} \epsilon_{i}(\mathbf{x})$. The energy density terms are related to the previous $\mu_{i}$ terms by the standard functional derivative $\mu_{i}(\mathbf{x})=\delta \epsilon_{i}(\mathbf{x}) / \delta n(\mathbf{x})$. The integrated energies are also reported in Table II.

We can now describe the vortex-nucleus configurations. For each zone we consider two cubic WS cells, adjacent along the $x$ direction, over which we will integrate the energies. The nuclear pinning configuration has the vortex axis along the $z$ direction and going through the center of one of the nuclei, the interstitial pinning configuration has the vortex equidistant between the two nuclei. For each configuration two possibilities arise: first we can find the surface $S_{M}$, coaxial with the vortex, where the pressure of the normal phase is equal to that of the superfluid one. Matter is normal inside $S_{M}$ and superfluid outside (the "mixed" phase), with the equality of pressures guaranteeing hydrostatic equilibrium. Alternatively, matter can always be superfluid (the "pure" phase), but with density going smoothly to zero along some other surface $S_{P}$ coaxial with the vortex. In Table III we give the energies for these two phases in both configurations when $\Delta=1 \mathrm{MeV}$; similar results are obtained for typical values $\Delta=0.5$ and $2 \mathrm{MeV}$. The energies are given as differences with the values for two vortex-free cells [10]. The last line gives the phase with minimum total energy, which will be chosen by the system as energetically convenient and used in the following calculations. Finally we evaluate the differences in energy between the nuclear pinning and the interstitial pinning configurations for each zone. These are reported term by term in Table IV for three values of the pairing gap.

TABLE III. Results for the "pure" $(P)$ and the "mixed" $(M)$ phases, calculated with a constant pairing gap $\Delta=1 \mathrm{MeV}$, in the nuclear pinning configuration and in the interstitial pinning configuration. The energies are reported as differences $\delta E_{i}=E_{i}-E_{0, i}$ between the energy with vortex and that without vortex, and they are given in MeV. The last line indicates the phase with minimum total energy for each zone.

\begin{tabular}{|c|c|c|c|c|c|c|c|c|}
\hline \multirow[t]{2}{*}{ Zone } & 1 & 2 & 3 & 4 & 1 & 2 & 3 & 4 \\
\hline & \multicolumn{4}{|c|}{ Nuclear pinning } & \multicolumn{4}{|c|}{ Interstitial pinning } \\
\hline$\delta E_{\mathrm{fer}}(P)$ & -188.0 & -231.4 & -379.7 & -481.6 & -6.0 & -60.5 & -191.2 & -307.9 \\
\hline$\delta E_{\mathrm{fer}}(M)$ & -5.0 & -26.8 & -42.4 & -18.0 & -5.2 & -27.4 & -44.1 & -18.5 \\
\hline$\delta E_{\mathrm{nuc}}(P)$ & 202.5 & 174.0 & 171.3 & 146.2 & 0.5 & 0.8 & 1.7 & 2.5 \\
\hline$\delta E_{\mathrm{nuc}}(M)$ & 0.4 & 0.5 & 0.5 & 0.3 & 0.5 & 0.8 & 1.7 & 0.6 \\
\hline$\delta E_{\mathrm{kin}}(P)$ & 100.8 & 126.8 & 213.2 & 272.1 & 3.7 & 34.8 & 110.4 & 177.2 \\
\hline$\delta E_{\mathrm{kin}}(M)$ & 3.1 & 15.8 & 22.3 & 2.5 & 3.4 & 16.4 & 23.7 & 2.8 \\
\hline$\delta E_{\mathrm{con}}(P)$ & 0.7 & 0.3 & 0.2 & 0.1 & 0.7 & 0.3 & 0.2 & 0.1 \\
\hline$\delta E_{\text {con }}(M)$ & 1.7 & 6.5 & 17.9 & 28.5 & 1.0 & 5.8 & 17.2 & 28.4 \\
\hline$\delta E_{\mathrm{tot}}(P)$ & 115.9 & 69.6 & 4.9 & -63.2 & -1.1 & -24.6 & -79.0 & -128.1 \\
\hline$\delta E_{\text {tot }}(M)$ & 0.2 & -4.1 & -1.7 & 13.3 & -0.3 & -4.4 & -1.6 & 13.4 \\
\hline Phase & $M$ & $M$ & $M$ & $P$ & $P$ & $P$ & $P$ & $P$ \\
\hline
\end{tabular}


TABLE IV. Energy differences $\Delta E_{i}=E_{i}^{(\mathrm{np})}-E_{i}^{(\mathrm{ip})}$ between the configuration with nuclear pinning and that with interstitial pinning, calculated with different constant values of the pairing gap $\Delta$.

\begin{tabular}{|c|c|c|c|c|c|c|c|c|c|c|c|c|}
\hline \multirow[t]{2}{*}{ Zone } & 1 & 2 & 3 & 4 & 1 & 2 & 3 & 4 & 1 & 2 & 3 & 4 \\
\hline & \multicolumn{4}{|c|}{$\Delta=0.5 \mathrm{MeV}$} & \multicolumn{4}{|c|}{$\Delta=1 \mathrm{MeV}$} & \multicolumn{4}{|c|}{$\Delta=2 \mathrm{MeV}$} \\
\hline$\Delta E_{\text {fer }}$ & 2.5 & 45.6 & -188.5 & -173.7 & 1.0 & 33.7 & 148.8 & 173.7 & -1.1 & 21.0 & 112.0 & -173.5 \\
\hline$\Delta E_{\text {nuc }}$ & -0.4 & -0.7 & 169.6 & 143.7 & -0.1 & -0.3 & -1.2 & 143.7 & 1.2 & 1.2 & 0.4 & 143.6 \\
\hline$\Delta E_{\text {kin }}$ & -1.4 & -26.9 & 102.8 & 94.9 & -0.6 & -19.0 & -88.1 & 94.9 & -0.4 & -11.9 & -64.5 & 94.8 \\
\hline$\Delta E_{\text {con }}$ & 0.7 & 5.8 & -0.003 & -0.001 & 1.1 & 6.2 & 17.7 & -0.005 & 3.3 & 8.5 & 19.5 & -0.01 \\
\hline$\Delta E_{\mathrm{tot}}$ & 1.5 & 24.2 & 84.0 & 64.9 & 1.3 & 20.5 & 77.3 & 64.9 & 3.0 & 18.8 & 67.5 & 64.8 \\
\hline
\end{tabular}

The results presented above suggest several comments. From Table III we see that the interstitial pinning configuration favors the pure phase all along the crust, while for the nuclear pinning configuration the type of phase depends on the density, the mixed one prevailing at lower densities. This is due to the different competition in the various phases and configurations between the kinetic and the internal (i.e., Fermi plus nuclear) terms. A similar trend is found also for different values of the pairing gap. Our calculations also show that the vortex cores, here defined by the surfaces $S_{M}$ and $S_{P}$, are over an order of magnitude smaller in the pure phase than in the mixed one, namely, the vortex cores can be empty of matter and very thin $(\sim 1 \mathrm{fm})$ or made of normal matter and quite large $(\sim 10 \mathrm{fm})$ [11]. Moreover, the dependence of the core on the local density is opposite in the two phases, with its radius increasing with $\rho$ in the mixed phase. From Table IV we deduce the main result of this paper, namely, that interstitial pinning takes place at all densities irrespective of the value of the pairing gap. The values for $\Delta E_{\text {tot }}$ get larger as one goes deeper into the crust. The crucial role of the internal energy contribution is quite evident, particularly for low densities: without taking it into account, one would find nuclear pinning in the outer zones. We also notice that had we considered only the mixed phases without internal energy, as it was done in all previous studies, we would have obtained the familiar result of nuclear pinning at large densities. We thus see how the inclusion in the model of different possible phases and of the internal energy terms, justified before on physical grounds, is fundamental in obtaining our conclusions.

The results we just described are definitely new and surprising, since they predict interstitial pinning all along the inner crust, while all previous studies found nuclear pinning for $\rho$ greater than $\sim 10^{13} \mathrm{~g} / \mathrm{cm}^{3}$. This could be crucial for any explanation of pulsar glitches based on depinning of vortices from the nuclear lattice. Indeed, it has been shown that interstitial pinning energies are orders of magnitude weaker than nuclear pinning energies [12], the former not being directly related to the energy differences between the two configurations (these, actually, turn out to be quite large in our model). Before drawing any conclusion, however, more detailed calcula- tions are needed to confirm our findings. Realistic nuclear potentials and density dependent pairing gaps should be used, even in the LDA. Of course, the final word will be given by a complete quantum calculation, which takes proximity effects into account. The present results, however, suggest to revisit the explanation of pulsar glitches in terms of very low pinning energies; whether this will improve the agreement between theory and observations, or rather make it impossible, is a fully open issue.

[1] P.W. Anderson and N. Itoh, Nature (London) 256, 25 (1975).

[2] M. A. Alpar, Astrophys. J. 213, 527 (1977).

[3] R. Epstein and G. Baym, Astrophys. J. 328, 680 (1988).

[4] P. M. Pizzochero et al., Phys. Rev. Lett. 79, 3347 (1997).

[5] J.W. Negele and D. Vautherin, Nucl. Phys. A207, 298 (1973).

[6] P. M. Pizzochero et al., Astrophys. J. 569, 381 (2002).

[7] Note that arguments based on local values for the energy, such as the one in Ref. [4], are not meaningful in selecting the physically relevant phase, which is a coherent property of the system and will thus depend on the global value of the energy.

[8] More exotic nuclear shapes ("pasta" or "lasagna" phases) could appear for densities above $\sim 10^{14} \mathrm{~g} / \mathrm{cm}^{3}$, deeper than the four zones considered here.

[9] The constant gravitational term ( $\left.\mu_{\text {grav }} \sim-10^{2} \mathrm{MeV}\right)$, which guarantees that the system is bound, has been included as a shift in the Fermi energy $\mu$, which therefore results positive.

[10] The fact that in some cases $\delta E_{\text {tot }}>0$ should not surprise: the existence of vortices follows from global macroscopic energy arguments, not on the local energy balance for a couple of cells.

[11] Note that the actual size of the tiny empty core obtained in the LDA for the "pure" phase is an artifact of the approximation, which is known to fail where the density goes to zero. When integrated over the system, however, the LDA results should be interpreted as indicating a superfluid density that decreases as one moves close to the vortex axis. This is consistent with the results obtained from the quantum Gross-Pitaevsky equation for an isolated vortex in a bosonic superfluid, which show a condensate decreasing to zero at the vortex axis.

[12] B. Link and R. Epstein, Astrophys. J. 373, 592 (1991). 